\title{
Lipid-polymer hybrid nanoparticles carrying doxorubicin and edelfosine combo for synergistic anticancer effect against drug-resistant Osteosarcoma
}

\author{
Ping Yang \\ The Eighth People's Hospital of Shanghai \\ Lian Zhang \\ The Eight People's Hospital of Shanghai \\ Tian Wang \\ The Eighth People's Hospital of Shanghai \\ Qi Liu \\ The Eighth People's Hospital of Shanghai \\ Jing Wang \\ The Eighth People's Hospital of Shanghai \\ Yaling Wang \\ The Eighth People's Hospital of Shanghai

\section{Zhiquan Tu} \\ The Eighth People's Hospital of Shanghai \\ Feng Lin ( $\sim$ kristisparksgks@yahoo.com ) \\ The Eighth People's Hospital of Shanghai
}

\section{Nano Express}

Keywords: Osteosarcoma, doxorubicin, edelfosine, nanoparticles, apoptosis, combination chemotherapy

Posted Date: March 4th, 2020

DOl: https://doi.org/10.21203/rs.3.rs-15987/v1

License: @ (i) This work is licensed under a Creative Commons Attribution 4.0 International License. Read Full License 


\section{Abstract}

In this study, we have prepared a novel folate receptor-targeted doxorubicin (DOX) and edelfosine (ET)loaded lipid polymer hybrid nanoparticle to enhance the anticancer efficacy in osteosarcoma. The dualdrug loaded nanoparticles showed a nanosized morphology and physiological stability. The targeted nanoparticles showed enhanced cellular internalization and subcellular distribution in MG63 cancer cells compared to that of non-targeted nanoparticles. Among many ratios of DOX and ET, 1:1 ratiometric combinations of drugs were observed to be highly synergistic in killing the cancer cells. MTT assay and caspase-3/7 activity assay clearly showed the superior anticancer efficacy of DE-FPLN formulations in inducing the cancer cell death. In vitro results indicate that the co-administration of two drugs in a folic acid-targeted nanoparticle could potentially induce the apoptosis and cell death. In vivo results displayed the potency of tumor cell killing and significant suppression of tumor growth without any detectable side effects. The lipid polymer hybrid nanocarriers with multiple properties of high drug loading, sequential and ratiometric drug release, improved physiological stability, prolonged blood circulation, and tumorspecific targeting are promising for the delivery of multiple drugs in the treatment of osteosarcoma.

\section{Introduction}

Osteosarcoma is one of the common forms of bone cancer accounting $60 \%$ of all bone cancer cases [1]. Osteosarcoma mainly affects children and adults of age been 10-18 years or within the first 2-decades of their life. It mainly originates from mesenchymal cells and largely presents in large bones such as femur or tibia [2]. Later on, primary osteo cells spread to lungs making it difficult to treat. The recent development in technology and diagnosis did not improve the 5 year-survival rate which is still $65 \%$ making it a big cause of concern. Current treatment options include surgery followed by chemotherapy. Several high doses of anticancer drugs such as doxorubicin, cisplatin, ifosfamide were used to treat osteosarcoma, however, none led to satisfactory cure or treatment $[3,4]$. The failure of chemotherapy mainly arises from drug resistance and acute side effects of high dose of anticancer drugs [5]. Therefore, a novel strategy needs to be developed to improve the survival rate and therapeutic efficacy in osteosarcoma.

Combination of chemotherapeutic drugs is adapted as a standard therapy in the clinic compared to single drug administration. The combination of anticancer drugs acts in multiple pathways and effectively kills the cancer cells compared to that of single drugs which often result in failure or lead to drug resistance. In this regard, doxorubicin (DOX) is an anthracycline analog widely used for the treatment of osteosarcoma as well as multiple other cancers such as breast cancer or leukemia [6]. Despite being one of the effective drug available in the market, its anticancer efficiency often hampered by the development of multidrug-resistance (MDR) that led to the progression of recurrence of cancer tissues. The main reason behind MDR is believed to be ATP-binding cassette transporters such as p-gp that pumps the drug out of the cancer cells [7]. Moreover, anthracyclines are substrate for p-gp and therefore responsible for MDR and failure in the chemotherapy process and hinders its application at the clinical level [8]. In order to reverse these limitations, combination of a second agent was considered an 
appropriate strategy. Edelfosine (ET) has been reported to exhibit synergistic therapeutic effect in osteosarcoma and has been reported to significantly decrease the dose of DOX administration [9]. The alkyl-lysophospholipid (ET) has been shown to efficacious in many cancer cells. The mechanism of action involved with ET involves binding the lipid rafts of plasma membrane inducing extrinsic apoptosis pathways $[10,11]$. Also it induces the apoptosis via mitochondria and endoplasmic reticulum $[12,13]$. Two different therapeutic targets of nucleus and membrane are expected to improve the therapeutic efficacy and minimize the adverse effects [14]. However, when administered in the free form, several drawbacks such as poor bioavailability, rapid clearance from the systemic circulations are reported.

Lipid-polymer hybrid nanoparticles (LPN) have recently emerged as a potent alternative to lipid or polymer nanoparticles separately [15]. LPN is typically polymeric nanoparticles embedded by biocompatible lipid layers. Both drugs (DOX and ET) are stably loaded in the hydrophobic PLGA and lipid structures [16]. The protective lipid layers effectively decrease the release of encapsulated drugs in systemic circulation and thereby reduce the side effects to normal cells [17]. The presence of polyethylene glycol (PEG) on the lipid shell improves the stability of particles and prevents the absorption of serum protein and thereby prolongs its systemic circulation $[18,19]$. These properties allow the accumulation of nanoparticles in the tumor tissues via a well-known enhanced permeability and retention (EPR) effect. In order to further increase the drug accumulation, targeting of nanoparticles to the tumor tissues would be beneficial [20]. In this regard, folate receptors (FR) which is overexpressed in the cancer cells binds specifically to the folic acid molecules [21]. The folate receptors are less expressed in the normal cells while overexpressed in the cancer cells and binds to folic acids in the nanoparticles [22].

The main aim of present study was to prepare DOX and ET-loaded lipid-polymer hybrid nanoparticles in order to increase the therapeutic efficacy in osteosarcoma. The dual-drugs were loaded in the LPN and characterized for wide range of in vitro and in vivo animal studies. The anticancer efficacy was tested in MG63 cancer cell-based xenograft tumor models. We have clearly showed that combination of DOX and ET significantly improved the anticancer efficacy.

\section{Conclusions}

In conclusion, we have prepared a novel folate receptor-targeted doxorubicin and edelfosine-loaded lipid polymer hybrid nanoparticle to enhance the anticancer efficacy in osteosarcoma. The dual-drug loaded nanoparticles showed a nanosized morphology and physiological stability. The targeted nanoparticles showed enhanced cellular internalization and subcellular distribution in MG63 cancer cells compared to that of non-targeted nanoparticles. Among many ratios of DOX and ET, 1:1 ratiometric combinations of drugs were observed to be highly synergistic in killing the cancer cells. MTT assay and caspase-3/7 activity assay clearly showed the superior anticancer efficacy of DE-FPLN formulations in inducing the cancer cell death. In vitro results indicate that the co-administration of two drugs in a folic acid-targeted nanoparticle could potentially induce the apoptosis and cell death. In vivo results displayed the potency of tumor cell killing and significant suppression of tumor growth without any detectable side effects. The lipid polymer hybrid nanocarriers with multiple properties of high drug loading, sequential and ratiometric 
drug release, improved physiological stability, prolonged blood circulation, and tumor-specific targeting are promising for the delivery of multiple drugs in the treatment of osteosarcoma.

\section{Materials And Methods}

\section{Synthesis of DOX/ET-loaded lipid-polymer targeted nanoparticles}

The dual-drug loaded lipid polymer nanoparticles were prepared by nanoprecipitation method. PLGA (10 $\mathrm{mg})$, DOX (1 mg) and ET (1 mg) were dissolved in acetonitrile and mixed together. Then lecithin, DSPEPEG (4:1 molar ratio) was dissolved in $5 \%$ ethanol aqueous solution at a weight ratio of $20 \%$ to that of PLGA mixture. The lipid fraction is heated at $65^{\circ} \mathrm{C}$ for $5 \mathrm{~min}$. The PLGA/acetonitrile solution was gradually added in the lipid solution a dropwise manner to allow self-assembly process. The organic solvents were gradually removed at $37^{\circ} \mathrm{C}$ via a rotary evaporation method. The thus-formed nanoparticle was washed thrice with distilled water using a centrifugal device with a molecular weight cutoff of 3500 $\mathrm{kDa}$. The nanoparticle was suspended in distilled water and stored in $4-8^{\circ} \mathrm{C}$ until further use. As much as possible, drug-loaded nanoparticles were prepared fresh for all the experiments. The drug-loading capacity and drug-loading efficiency were calculated using the same centrifugal tubes. The filtrate containing the free drug was evaluated by HPLC method and plotted.

\section{In vitro drug release study}

The drug release of DOX and ET from DE-LN and DE-FLN was studied using a dialysis tube (Spectra/Pore, MWCO 3500). The study was performed at $37^{\circ} \mathrm{C}$ in a regular shaker (100 rpm). The dual drug-loaded nanoparticle dispersions were sealed in a dialysis tube and placed in a $30 \mathrm{ml}$ of acetate buffered saline (ABS, pH 5.0) and phosphate buffered saline (PBS, pH 7.4) in a Falcon tube. The tube was placed in a shaker bath at a speed of $100 \mathrm{rpm} / \mathrm{min}$ and maintained at $37^{\circ} \mathrm{C}$. At predetermined time, small aliquots of samples were collected and replaced with equal volume of the fresh sample to maintain the sink conditions. The amount of drug released in the buffer was calculated using HPLC method. The HPLC system (Waters, Milford, MA, USA) consists of Waters 1525 Binary HPLC pump, a Waters 2707 Autosampler, and a Waters 2475 multi $\lambda$ fluorescence detector. A column consists of C-18 column (150 $\mathrm{mm} \times 4.6 \mathrm{~mm}, 5 \mu \mathrm{m}$ particle size, and $100 \AA$ pore size, AkzoNobel/Kromasil, Brewster, NY, USA) was used at a room temperature. Flow rate was maintained at $1 \mathrm{ml}$ per minute.

\section{In vitro cell culture}

MG63 osteosarcoma cells were cultured in RPMI- 1640 growth medium supplemented with $10 \%$ FBS and $1 \%$ antibiotic mixture. The cells were grown in ambient conditions of $5 \%$ carbon dioxide and moisture status.

\section{In vitro cellular uptake}

The targeting ability of nanoparticles towards MG63 cells were studied by confocal laser scanning microscopy (CLSM). The 6-well plate was covered with cover slip and cells were seeded in the capacity of 
$2 \times 10^{5}$ cells per well and incubated for $12 \mathrm{~h}$. Next day, cells were exposed with LPN-loaded with fluorescent Nile Red at a concentration of $0.25 \mathrm{mg} / \mathrm{ml}$ and incubated for $2 \mathrm{~h}$. The cells were washed and fixed with $4 \%$ paraformaldehyde and incubated for $10 \mathrm{~min}$ followed by staining with DAPI solution for $5 \mathrm{~min}$. The cells were well-rinsed with PBS and observed under confocal microscope (Zeiss LSM 710; Carl Zeiss Meditee $A G)$.

\section{In vitro cytotoxicity assay}

The in vitro cytotoxicity assay in MG63 cancer cell was evaluated by MTS as per the guidelines of manufacturers of CellTiter $96^{\circledR}$ Aqueous One Solution Cell Proliferation Assay (Promega, Madrid, Spain). In brief, 8000 cells were seeded in the each well of 96 -well plate and incubated for $24 \mathrm{~h}$. After that, cells were treated with different concentrations of free drugs and free dru combination and drug-loaded nanoparticles and incubated for $24 \mathrm{~h}$. After $24 \mathrm{~h}$, supernatant and all media was removed and washed twice with PBS. The cells were then added with $100 \mu$ l of complete media containing $15 \mu$ l of MTS solution and incubated for $4 \mathrm{~h}$. The absorbance was measured using a microplate reader (iEMS reader MF, Labsystem, Helsinki, Finland) at a wavelength of $690 \mathrm{~nm}$. The IC50 value calculations and statistical analysis was performed using GraphPad Prism software. The combination index was calculated using CalcuSyn software (Biosoft, Cambridge, UK). A Cl value of less than 0.9 indicates synergism while 0.9-1.1 indicates additive and anything above 1.1 indicates the antagonistic characteristics.

\section{Caspase $3 / 7$ activity}

The caspase-3/7 activity was evaluated using a Caspase-Glo-3/7 assay kit (Promega, USA) as per the manufacturer's protocol. In brief, 8000 cells were seeded in the each well of 96 -well plate and incubated for $24 \mathrm{~h}$. After that, cells were treated with different concentrations of free drugs and free drug combination and drug-loaded nanoparticles and incubated for $24 \mathrm{~h}$. After $24 \mathrm{~h}$, supernatant and all media was removed and washed twice with PBS. The caspase activity was measured by adding the assay reagent in a proportion of 1:1 to each well. The luminescence signal was measured using an Tecan GENios microplate reader (Tecan Group Ltd, Switzerland) after incubating for 30 min post reagent addition. The experiments were performed in triplicate.

\section{Pharmacokinetic analysis of DE-FPLN}

Sprague-Dawley rats (male) weighing $200 \pm 10 \mathrm{~g}$ were divided into four groups of 5 rats (DOX, EDL, DEFPLN (DOX) and DE-FPLN (EDL). The animals were caged in an animal house maintained at $25^{\circ} \mathrm{C}$ and $60 \% \mathrm{RH}$. All the animal studies are performed in compliance with the Institutional Animal Ethical Care and Management (IAECM) guidelines, Luoyang Orthopedic Hospital of Henan Province, China. The animals were received free DOX, free EDL, and DE-FPLN (DOX/EDL) by intravenous administration at a fixed dose of $5 \mathrm{mg} / \mathrm{kg}$. Free DOX and EDL were dissolved in PEG 400 and administered at a dose of $5 \mathrm{mg} / \mathrm{kg}$. In case of DE-FPLN, the lyophilized powder was dissolved in physiological saline $(0.9 \% \mathrm{NaCl})$. Blood samples $(300 \mu \mathrm{L})$ were collected from the right femoral artery at pre-determined times $(0.25,0.5,1,2,4,8$, 12 and $24 \mathrm{~h}$ ) after administration of these formulations. The samples were collected in heparin- 
containing tubes $(100 \mathrm{lU} / \mathrm{mL})$ and then immediately centrifuged (Eppendorf, Hauppauge, NY, USA) at $12,000 \mathrm{rpm}$ for $10 \mathrm{~min}$. The plasma supernatant was collected and stored at $-20 \circ \mathrm{C}$ until further analysis. The DOX/EDL was extracted after the precipitation of unwanted protein. $150 \mu \mathrm{L}$ of plasma was mixed with $150 \mu \mathrm{L}$ of acetonitrile for $30 \mathrm{~min}$. The samples were then centrifuged at 12,000 rpm for $10 \mathrm{~min}$ and $10 \mu \mathrm{L}$ of the supernatant was injected into the HPLC system for DOX and EDL analysis.

\section{In vivo antitumor efficacy}

The BALB/c female mice with an average weight ranging from 20-22 gram were purchased and given humanely care with free access to food and water. All the animal studies are performed in compliance with the Institutional Animal Ethical Care and Management (IAECM) guidelines, The Eighth People's Hospital of Shanghai, China. In brief, MG63 cells were grown in the culture medium and after $80 \%$ confluence; cells were harvested from the culture flask and subcutaneously implanted in the right flank of the mice in the capacity of $5 \times 10^{6}$ cells/mouse. The tumor growth was closely observed and the experiment was initiated when the tumor volume reaches approximately $100 \mathrm{~mm}^{3}$. The mice were divided into 6 groups with 8 mice in each group. The mice groups were administrated with free DOX, ET, DOX/ET cocktail, DE-PLN and DE-FPLN at a maximum dose of $5 \mathrm{mg} / \mathrm{kg}$ of mice body eight for 3 times. All mice were tagged and tumor volumes were measured at predetermined time interval using the tumor volume (V) calculation formula $V=a \times b \times 1 / 2$ whereas ' $a$ ' and ' $b$ ' are respective long and short diameters of the tumor. The tumor size was measured using an Vernier caliper.

\section{Histological analysis of tumors}

The tumors were surgically removed from the mice after sacrificing it humanely following all the animal handling protocols. The tumors were immediately fixed in $10 \%$ formalin for $2 \mathrm{~h}$ and embedded in a paraffin in a thickness of $5 \mathrm{~mm}$. The tissue section is then subjected to H\&E staining and observed through the fluorescence microscope (Olympus, NIKON).

\section{Statistical analysis}

All data are performed as the mean \pm standard deviation. All statistical analysis was performed using SPSS 10.0 software (SPSS Inc., Chicago, USA). A statistical difference of $p<0.05$ was considered significant.

\section{Results And Discussion}

\section{Formulation and optimization of DOX/ET-loaded folate-targeted lipid polymer nanoparticles}

The schematic presentation of preparation of DOX/ET-loaded lipid polymer hybrid nanoparticle is presented in Figure 1. The DOX and ET along with PLGA were dissolved in organic solvent and stirred. The lipid mixture was added to the polymeric solution and allowed to form the self-assembly resulting in the creation of lipid-polymer hybrid nanoparticles. The hydrophobic nature of the drugs allowed the stable 
incorporation in the lipid matrix and hydrophobic PLGA. Earlier, studies have shown that the presence of PEG on the outer surface will ensure the protection from reticuloendothelial (RES) based clearance system that could potentially result in the prolonged blood circulation and reduced non-specific binding. The presence of folic acid moiety will confer the targeting capacity to the nanoparticles towards the tumors tissues in the body.

The particle size and surface charge characteristics were evaluated by dynamic light scattering (DLS) analysis. The mean particle size of DE-FPLN was observed to be $122.5 \pm 2.45 \mathrm{~nm}$ with a narrow distribution index of 0.140 (Figure 2a). The average surface charge of DE-FPLN was observed to be $-18.4 \pm 1.26 \mathrm{mV}$. The particle size was further confirmed by the TEM imaging (Figure $2 \mathrm{~b}$ ). All the particles perfectly spherical in shape and uniformly spread over the copper grid. Especially, a greyish shell was observed at the periphery of each particle that might be due to the presence of lipid shell. A particle in the nanosize ranges deemed suitable for the tumor targeting as it can penetrate the tumor tissues via enhanced permeation and retention (EPR) property. It is reported and wide recognized that tumors possess leaky vasculature coupled with poor drainage access which allows the high accumulation of particles and intracellular concentrations when the particles are nanosized [22]. The loading capacity of DOX in DE-FPLN was $6.89 \% \mathrm{w} / \mathrm{w}$ while ET was $5.75 \% \mathrm{w} / \mathrm{w}$, respectively.

\section{In vitro drug release}

The release of DOX and ET from DE-FPLN under different $\mathrm{pH}$ conditions was evaluated by dialysis method. As seen (Figure 2c), differential release pattern was observed under physiological and acidic $\mathrm{pH}$ conditions. The release rate of drugs was higher in acidic conditions compared to that of in $\mathrm{pH} 7.4$ conditions. For example, $18 \%$ of DOX released in $24 \mathrm{~h}$ in $\mathrm{pH} 7.4$ conditions compared to $~ 32 \%$ in $\mathrm{pH} 5.0$ conditions. Similarly, $12 \%$ of ET released in $24 \mathrm{~h}$ in $\mathrm{pH} 7.4$ conditions compared to nearly $25 \%$ ET release in $\mathrm{pH} 5.0$ conditions. It should also be noted that release rate of DOX was relatively higher than that of the release rate of ET in both $\mathrm{pH}$ conditions. A differential release pattern for both the drugs might be attributed to the encapsulation of one drug in polymer core and another in lipid matrix or vice versa. Importantly, both the drugs showed a sustained release of drug from the nanoparticles. The release trend continued until the end of the study period with higher release in acidic media mimicking the tumor microenvironment. This type of sequential release of drugs in the tumor cells results in effective synergistic therapeutic effects. Besides, enhanced drug release in lower $\mathrm{pH}$ conditions compared to that in physiological conditions boosts the prospect of higher drug release in the lysosomal/endosomal pH conditions in the tumors [23].

\section{Intracellular uptake in MG63 osteosarcoma cells}

The targeting ability of the nanoparticles (DE-PLN and DE-FPLN) in MG63 cancer cell was evaluated by confocal laser scanning microscopy (Figure 3). CLSM images showed a red fluorescence from Nile redloaded nanoparticles in the lysosomal regions of cancer cells. Data clearly reveal that folic acid-targeted nanoparticle (DE-FPLN) showed a remarkably stronger red fluorescence compared to that of non-targeted DE-PLN indicating that the receptor-mediated internalization via FR-mediated endocytosis might be 
responsible for enhanced accumulation of particles. FR targeting played a seemingly crucial role in enhancing the cellular uptake of nanoparticles by MG63 cells. Accumulation of non-targeted nanoparticles in the cancer cells further highlight the fact that other mechanisms such as clathrin or caveolae-mediated could be associated with the nanoparticles uptake. It is believed that nanoparticles enter the acidic lysosomes and the nanoparticles get destabilized and results in the release of encapsulated drugs. These drugs then reach their targeted location inside the tumors. The cellular internalization of nanoparticles was further confirmed after pretreatment with free folate. As shown, marked reduction in cellular uptake of DE-FPPLN was observed in cells pretreated with free folate indicating the expression of folate receptors and receptor-mediated cell uptake in the cancer cells (Figure S1).

\section{In vitro cell viability - Combination Index}

Administration of single drugs often resulted in poor efficacy and multidrug resistance (MDR). In this regard, combination chemotherapy has been proven to be remarkably effective than single drugs. The use of multiple drugs will act on the different pharmacological pathways and prevent the drug resistance and improve the therapeutic efficacy. It is well known that specific ratio of drugs is particularly more effective while certain ratios are ineffective. In order to evaluate the perfect ratio of two drugs, drugs were combined in different ratiometric combination and cell viability was evaluated. As seen (Figure 4a), different ratiometric combinations have different effect on the cell viability of cancer cells. The therapeutic effect of combinations of drugs are evaluated in terms of synergistic, additive and antagonistic. The Chou and Talalay method-based $\mathrm{Cl}$ tells that the values below $<0.9$ indicates the drug synergy while $\mathrm{Cl}$ between 0.9-1.1 indicates additive nature and $>1.1$ indicates the antagonistic effect of the drugs. As seen, DOX:ET were highly synergistic in 1:1 w/w ratio indicating a maximal synergistic interaction between the drugs. The DOX:ET at 1:2 and 2:1 though synergistic, it was less compared to that of 1:1 ratiometric combinations. On the contrary, drug ratios of 5:1 and 10:1 were antagonistic in nature. Based on the $\mathrm{C} 1$ value, we have chosen DOX:ET to study further for all the in vitro and in vivo studies.

\section{In vitro cytotoxicity assay}

Following the ratiometric analysis of DOX and ET, in vitro cytotoxic effect of free drugs and drug-loaded nanoparticles (targeted and non-targeted) was evaluated in MG63 osteosarcoma cells. As seen (Figure $4 \mathrm{~b})$, all the formulations showed a typical dose-dependent cytotoxic effect in the cancer cells. The cell viability of cocktail DOX:ET (DE) treated cells were significantly lesser compared to that of single drugs either DOX or ET alone further suggesting potential of combination of drugs. It should be noted that dualdrug loaded nanoparticle showed relatively higher anticancer effect than the free combination of drugs. Importantly, folate receptor-targeted DE-FPLN showed a remarkable anticancer effect in the MG63 cancer cells. The cytotoxicity profiles were fitted in logistic model to calculate the IC50 value - a concentration required to kill $50 \%$ of the cancer cells. The IC50 values of DOX, ET, DE, DE-PLN and DE-FPLN stood at 4.5 $\mu \mathrm{g} / \mathrm{ml}, 6.4 \mu \mathrm{g} / \mathrm{ml}, 2.3 \mu \mathrm{g} / \mathrm{ml}, 1.08 \mu \mathrm{g} / \mathrm{ml}$, and $0.26 \mu \mathrm{g} / \mathrm{ml}$, respectively. Data clearly reveal the superior 
anticancer potential of DE-FPLN formulation in controlling the proliferations of cancer cells. DE-PLN though showed notable decrease in cell viability due the combination therapeutics, DE-FPLN showed the significantly lower cell viability attributed to the higher cellular internalization of targeting ligand-based carrier system.

\section{Caspase $-3 / 7$ based apoptosis activity}

The mechanism of cell death was evaluated in terms of cell apoptosis using caspase-3/7 activity assay in MG63 cells. For this purpose, MG63 cells were exposed with different formulations and incubated for 24h. As seen (Figure 5a), caspase-3/7 activity of cocktail DE remarkably increased compared to that of single drug treatment. Consistent with the cell viability assay, DE-PLN showed higher caspase-3/7 activity than that of free drug cocktails. Interestingly, DE-FPLN showed 1.5-fold and 3-fold higher caspase-3/7 activity compared to that of DE-PLN and DE cocktail, indicating the co-administration of two drugs in a folic acid-targeted nanoparticle could potentially induce the apoptosis and cell death. Such enhancing the cytotoxic activity between DOX and ET has been reported in Ewing's sarcoma cells that could be attributable due to different mechanism of actions [24]. DOX is reported to exert its pharmacological effect by the inhibition of DNA synthesis in nucleus while ET targets the cell membrane, mitochondria and endoplasmic reticulum $[25,26]$.

\section{Biodistribution and Pharmacokinetic analysis}

A biodistribution study of Cy5.5-based DE-PLN and DE-FPLN was performed in xenograft animal model following the i.v. administration. As shown by whole body image and ex vivo organ image, DE-FPLN exhibited preferential accumulation in tumors compared to that of DE-PLN indicating the tumor targeting ability of DE-FPLN (Figure S2). A notable accumulation of DE-PLN in the tumor might be attributed to the passive accumulation by EPR effect, however, relatively lower fluorescence intensity was observed compared to that of DE-FPLN. It must be emphasized that vital organs including heart, kidney and lungs pretty much showed similar levels of nanoparticle uptake.

The plasma concentration-time profiles of DOX and EDL following single dose intravenous (IV) administration of free form and nanoparticle encapsulated form are presented in Figure 5b. Free DOX, EDL and DE-FPLN (DOX/EDL) were administrated at a dose of $10 \mathrm{mg} / \mathrm{kg}$ via the femoral vein. Free DOX and EDL were rapidly cleared from the circulation within $4 \mathrm{~h}$ of IV administration and exhibited linear pharmacokinetics. As reported previously, the linear pharmacokinetic profile of DOX remained the same whether administered at high or low dose. In contrast, DOX and EDL had a remarkably prolonged plasma circulation time after administration of DE-FPLN (DOX/EDL) and maintained a therapeutic drug level throughout the study period. For example, $0.121 \pm 0.25 \mu \mathrm{g} / \mathrm{ml}$ of free DOX was observed after $4 \mathrm{~h}$ of i.v administration whereas $0.256 \pm 0.21 \mu \mathrm{g} / \mathrm{ml}$ of DOX was observed from nanoparticle formulation after $24 \mathrm{~h}$ indicating the superior potential of carrier-mediated delivery system. The prolonged blood circulation profile of DE-FPLN was mainly attributed to the shielding effect of PEG, the excellent stability of the carrier/drug formulation in blood circulation, and the negative surface charge of the nanoparticles. Additionally, nanosized particles might be able to evade the macrophage system in the systemic blood 
circulation. The presence of a PEG outer shell in the micelles reduced the effective surface charge and increased the hydrophilicity that would decrease the liver and splenic uptake.

\section{In vivo antitumor efficacy}

The therapeutic efficacy of formulations was evaluated by the intravenous administration for formulations in tumor-bearing mice model. The mice were administered with DOX, ET, DE, DE-PLN and DE-FPLN, respectively. As shown (Figure 6a), free DOX and ET did not have much effect on the tumor growth while cocktail combination DE showed obvious tumor regression compared to single treatment and control. Importantly, DE-FPLN outperformed all the other groups and significantly delayed the tumor progression in the animal models. DE-FPLN showed 1.5-fold lower tumor volume than DE-PLN while it showed 2-fold lower tumor volume and 3-fold lower tumor volume for DE and single drugs, respectively. The main reason behind the excellent antitumor efficacy might be due to prolonged blood circulation and active targeting of the nanoparticles to the tumor tissues. The folate receptors overexpressed in the tumors will bind specifically to the folic acid ligand conjugated on the nanoparticle surface and results in enhanced accumulation in the local area and high therapeutic efficacy. Significant differences in tumor volume were observed between mice treated with $2.5 \mathrm{mg} / \mathrm{kg}$ and $5 \mathrm{mg} / \mathrm{kg}$, however, insignificant difference was observed at higher doses (Figure 6b). Except at the last time point, mice treated with 5 $\mathrm{mg} / \mathrm{kg}$ and $10 \mathrm{mg} / \mathrm{kg}$ was insignificant. The experiment further reiterates the fact that $5 \mathrm{mg} / \mathrm{kg}$ of the combination of DOX and EDL could be appropriate. Besides, hallmarks of Tumors are characterized by the presence of poor lymphatic drainage and leaky vasculature that allows the internalization of nanocarriers sized up to $\sim 200 \mathrm{~nm}$ specifically into the tumoral regions, commonly referred to as EPR effect. The features of improved physiological stability and prolonged blood circulation contributed to selective accumulation and long-term retention of DE-FPLN at the tumor sites and enhanced therapeutic efficacy. Fernandez et al, have reported a synergistic effect of DOX and EDL. The authors reported that the synergistic effect was well-correlated with Caspase-3/7 activity indicating a caspase-mediated cell death [27]. Other authors also observed the combination effect of DOX and EDL in Ewing's sarcoma cells [28]. DOX is known to exert its effect by inhibition of DNA synthesis in nucleus, while, EDL targets the membrane of the cells, endoplasmic reticulum and mitochondria. Stability and suitability of carriers also played an important role in enhancing the anticancer effect of encapsulated drugs. For example, Zhang et al reported the enhanced anticancer effect employing similar polymer-lipid hybrid nanoparticles [29]. Authors have reported that polymer-lipid hybrid nanoparticles improved the pharmacokinetic profile of dual drugs (DOX and Mitomycin C) and enhanced the antitumor effect in xenograft breast cancers.

H\&E analysis revealed that DE-FPLN exerted remarkable damage to the tumor tissues compared to any other administered groups (Figure 6c). DE-FPLN treated tumors exhibited pyknotic cells in which nuclei were condensed which are seemingly signs of apoptosis and dead cells. Furthermore, mice treated with DE-FPLN did not show any significant changes in the body weight throughout the study period indicating a lack of any toxic effects. In contrast, mice treated with free DOX and DE resulted in $10 \%$ shedding of body weight which is consistent with the other reports of toxicity (Figure 6d). In case of DE-FPLN, low dosage of both drugs which is stably incorporated in the nanoparticles was beneficial in reducing the side 
effects and highlights the superior advantages of nanocarrier system. The significant improvement in the antitumor efficacy stem from the fact that DE-FPLN delivers the drug to the tumor tissues in a specific ratiometric manner that allows the synergistic killing of the cancer cells and tumor regression. In contrast, free drug and cocktail combinations (DE) may not equally penetrate the tumors and results in subpar therapeutic effect.

\section{Acute toxicity analysis}

Drug-related toxicity to major organs was evaluated by H\&E staining. Free DOX treatment to the animals resulted in serious damage to the liver, kidney and heart (Figure 7). EDL was relatively less toxic compared to that of DOX as observed from the H\&E image. Hepatic lesions were observed with severe atrophy of hepatic cells. The free DOX resulted in acute cellular swellings of the liver and the mean hepatocyte diameter for the free DOX-treated group was $26.18 \pm 2.16 \mu \mathrm{m}$ compared to $14.25 \pm 1.68 \mu \mathrm{m}$ in the non-treated animal group. Cardiac H\&E revealed that free DOX resulted in focal rarefactions, focal areas of disrupted cardiac muscle fibers and cytoplasmic vacuolization. In contrast, DE-FPLN showed no myocardial degeneration and was devoid of any cardiotoxicity. The kidney in DE and DOX-treated group showed notable congestion with marked degenerative changes, and disrupted epithelium. The sections of liver in DE and DOX-treated group showed marked central vein congestion, marked bile duct hyperplasia, and dilation of sinusoidal spaces, and some sections showed marked degenerative changes. The H\&E stained sections of liver and kidney from formulation-treated group appeared normal suggesting lack of any hepatotoxicity or nephrotoxicity. No such damage or sign of toxicity was observed in DE-FPLN treated animal group suggesting the efficacy of carrier-based drug delivery. The ability of the carrier system to decrease the organ damage and increase the anticancer efficacy is of great significance.

Despite the excellent finding in this investigation, study is not without the limitations. It was assumed in this study that a higher dose would confer greatest efficacy, however, in terms of toxicity, maximum tolerable dose (MTD) is not determined. The acute toxicity effects monitoring performed in the current study was done only at the dose range tested, but not at the MTD of the various compounds involved (as the MTDs have not been determined). Future studies will focus on quantifying dose-response effects, with simultaneous emphasis on MTD. Furthermore, we would like to continue the study with different targeting ligand or combination of two or more ligands.

\section{Abbreviations}

DOX

Doxorubicin

ET

Edelfosine

PLN

Polymer-lipid hybrid nanoparticles

DE-FPLN 
DOX/ET-loaded folic acid conjugated PLN

EPR

Enhanced permeation and retention effect

FR

Folate receptors

MDR

Multidrug resistance

\section{Declarations}

\section{Author's contributions}

All authors contributed equally to the entire research project and all authors approved the final version of present manuscript.

\section{Funding}

This study was supported by Xuhui District Medical Science and Technology Project (Grant number: SHXH201736 and SHXH201709).

\section{Availability of Data and Materials}

All data generated or analyzed during this study are included in this published article and its supplementary information files.

\section{Competing Interest}

The authors declare no competing financial interest.

\section{Author details}

Ping Yang, Lian Zhang, Tian Wang, Qi Liu, Jing Wang, Yaling Wang, Zhiquan Tu, Feng Lin*

Department of Oncology, The Eighth People's Hospital of Shanghai, Shanghai, 200233, China

\section{Acknowledgement}

N/A

\section{References}

1. Kansara M, Teng MW, Smyth MJ, Thomas DM. Translational biology of osteosarcoma. Nat Rev Cancer 2014;14:722-735.

2. Botter SM, Neri D, Fuchs B. Recent advances in osteosarcoma. Curr Opin Pharmacol. 2014;16:15-23. 
3. Ferrari S, M Smeland, F Mercuri, A Bertoni, P Longhi, P Ruggieri et al. Neoadjuvant chemotherapy with high-dose ifosfamide, high-dose. methotrexate, cisplatin, and doxorubicin for patients with localized osteosarcoma of the extremity: a joint study by the Italian and Scandinavian Sarcoma Groups. J Clin Oncol. 2005;23:8845-8852.

4. Schwartz CL, Gorlick R, Teot L, Krailo M, Chen Z, Goorin A, et al. Multiple drug resistance in osteogenic sarcoma: INT0133 from the Children's Oncology Group. J Clin Oncol. 2007;25:20572062.

5. Siegel R, Naishadham D, Jemal A. Cancer statistics, 2013. CA Cancer J Clin. 2013;63:11-30.

6. Ramasamy T, Ruttala HB, Sundaramoorthy P, Poudel BK, Youn YS, Ku SK, Choi HG, Yong CS, Kim JO. Multimodal selenium nanoshell-capped Au@ mSiO 2 nanoplatform for NIR-responsive chemophotothermal therapy against metastatic breast cancer. NPG Asia Materials 2018;10:197-216.

7. Tran TH, Ramasamy T, Choi JY, Nguyen HT, Pham TT, Jeong JH, Ku SK, Choi HG, Yong CS, Kim JO. Tumor-targeting, $\mathrm{pH}$-sensitive nanoparticles for docetaxel delivery to drug-resistant cancer cells. Int $\mathrm{J}$ Nanomedicine. 2015;10:5249-62.

8. Thorn CF, Oshiro C, Marsh S, Hernandez-Boussard T, McLeod H, Klein KE, et al. Doxorubicin pathways: pharmacodynamics and adverse effects. Pharmacogenet Genomics 2011;21:440-446.

9. Bonilla X, Dakir E, Mollinedo F, Gajate C. Endoplasmic reticulum targeting in Ewing's sarcoma by the alkylphospholipid analog Edelfosine. Oncotarget 2015;6:14596-14613.

10. Yao C, Wei JJ, Wang ZY, Ding HM, Li D, Yan SC et al. Perifosine induces cell apoptosis in human osteosarcoma cells: new implication for osteosarcoma therapy? Cell Biochem Biophys. 2013;65:217227.

11. Gonz alez-Fernandez Y, Zalacain M, Imbuluzqueta E, Sierrasesumaga L, Patino-García A, BlancoPrieto M. Lipid nanoparticles enhance the efficacy of chemotherapy in primary and metastatic human osteosarcoma cells. J Drug Deliv Sci Technol. 2015;30:435-442.

12. Estella-Hermoso de Mendoza A, Preat V, Mollinedo F, Blanco-Prieto MJ. In vitro and in vivo efficacy of edelfosine-loaded lipid nanoparticles against glioma. J Control Release 2011;156:421-426.

13. Mollinedo F, Fernandez-Luna JL, Gajate C, Martin-Martin B, Benito A, Martinez-Dalmau R. Selective induction of apoptosis in cancer cells by the ether lipid ET-18-OCH3 (Edelfosine): molecular structure requirements, cellular uptake, and protection by Bcl-2 and Bcl-X(L). Cancer Res. 1997;57:1320-1328.

14. Gajate C, Mollinedo F. Biological activities, mechanisms of action and biomedical prospect of the antitumor ether phospholipid ET-18-OCH(3) (edelfosine), a proapoptotic agent in tumor cells. Curr Drug Metab. 2002;3:491-525

15. Ramasamy T, Ruttala HB, Gupta B, Poudel BK, Choi HG, Yong CS, Kim JO. Smart chemistry-based nanosized drug delivery systems for systemic applications: A comprehensive review. J Control Release 2017;258:226-253.

16. Hadinoto K, Sundaresan A, Cheow WS. Lipid-polymer hybrid nanoparticles as a new generation therapeutic delivery platform: a review. Eur J Pharm Biopharm. 2013;85:427-443. 
17. Li Y, Wu H, Yang X, Jia M, Li Y, Huang Y, Lin J, Wu S, Hou Z. Mitomycin C-soybean phosphatidylcholine complex-loaded self-assembled PEG-lipid-PLA hybrid nanoparticles for targeted drug delivery and dual-controlled drug release. Mol Pharm. 2014;11:2915-2927.

18. Ramasamy T, Ruttala HB, Chitrapriya N, Poudel BK, Choi JY, Kim ST et al. Engineering of cell microenvironment-responsive polypeptide nanovehicle co-encapsulating a synergistic combination of small molecules for effective chemotherapy in solid tumors. Acta Biomater. 2017;48:131-143.

19. Thevenot J, Troutier AL, David L, Delair T, Ladavière C. Steric stabilization of lipid/polymer particle assemblies by poly(ethylene glycol)-lipids. Biomacromolecules. 2007;8:3651-3660.

20. Salvador-Morales C, Zhang L, Langer R, Farokhzad OC. Immunocompatibility properties of lipidpolymer hybrid nanoparticles with heterogeneous surface functional groups. Biomaterials 2009;30:2231-2240.

21. Ai JW, Liu B, Liu WD. Folic acid-tagged titanium dioxide nanoparticles for enhanced anticancer effect in osteosarcoma cells. Mater Sci Eng C Mater Biol Appl. 2017;76:1181-1187.

22. Montazerabadi A, Beik J, Irajirad R, Attaran N, Khaledi S, Ghaznavi H, Shakeri-Zadeh A. Folatemodified and curcumin-loaded dendritic magnetite nanocarriers for the targeted thermochemotherapy of cancer cells. Artif Cells Nanomed Biotechnol. 2019;47(1):330-340

23. Brannon-Peppas L, Blanchette JO. Nanoparticle and targeted systems for cancer therapy. Adv Drug Deliv Rev. 2004;56:1649-1659.

24. Meng F, Cheng R, Deng C, Zhong Z. Intracellular drug release nanosystems. Mater Today 2012;15:436-442.

25. Bonilla X, Dakir E, Mollinedo F, Gajate C. Endoplasmic reticulum targeting in Ewing's sarcoma by the alkylphospholipid analog Edelfosine. Oncotarget 2015;6:14596-14613

26. Gajate C, Mollinedo F. Lipid rafts, endoplasmic reticulum and mitochondria in the antitumor action of the alkylphospholipid analog Edelfosine. Anticancer Agents Med Chem. 2014;14:509-527

27. González-Fernández Y, Imbuluzqueta E, Zalacain M, Mollinedo F, Patiño-García A, Blanco-Prieto MJ. Doxorubicin and edelfosine lipid nanoparticles are effective acting synergistically against drugresistant osteosarcoma cancer cells. Cancer Lett. 2017;388:262-268.

28. Bonilla X, Dakir E, Mollinedo F, Gajate C. Endoplasmic reticulum targeting in Ewing's sarcoma by the alkylphospholipid analog Edelfosine. Oncotarget 2015;6:14596-14613

29. Zhang RX, Cai P, Zhang T, Chen K, Li J, Cheng J et al. Polymer-lipid hybrid nanoparticles synchronize pharmacokinetics of co-encapsulated doxorubicin-mitomycin $\mathrm{C}$ and enable their spatiotemporal codelivery and local bioavailability in breast tumor. Nanomedicine. 2016;12: 1279-90.

\section{Figures}




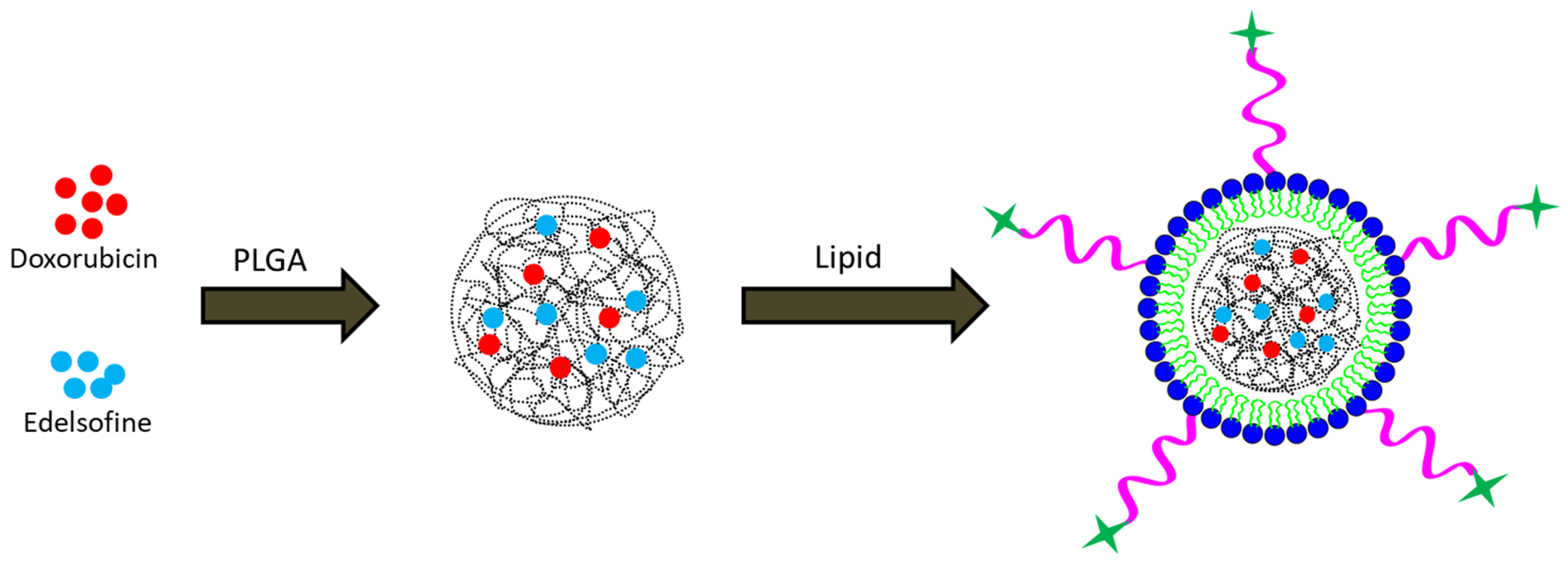

Folate-conjugated lipid/polymer hybrid nanoparticles

\section{Figure 1}

Schematic illustration of formulation design. Schematic presentation of preparation of DOX and ETloaded folic acid targeted lipid polymer hybrid nanoparticles. 
(a)

(b)
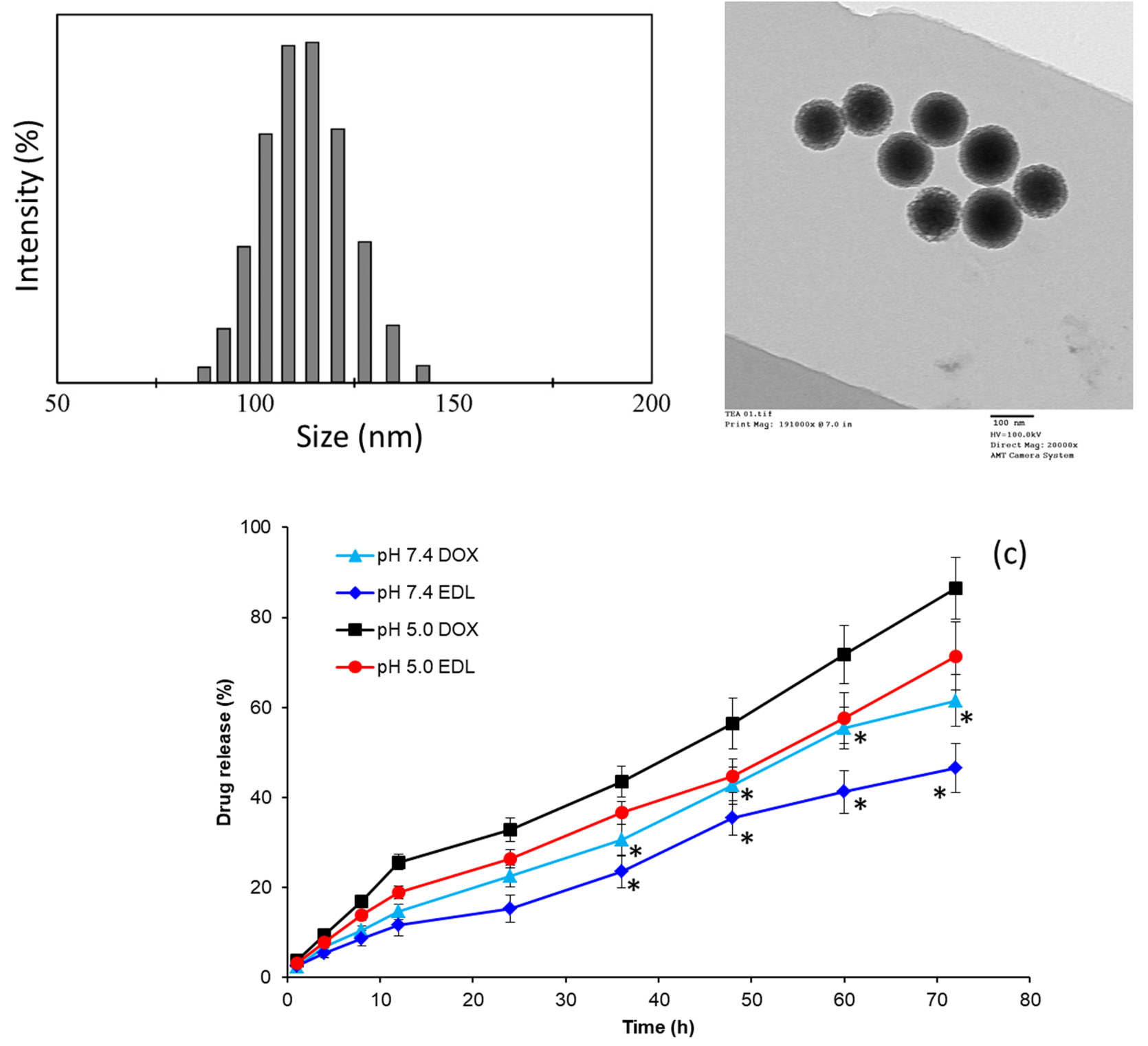

Figure 2

Physicochemical characterization of nanoparticles. (a) Particle size distribution of DE-FPLN by dynamic light scattering (DLS) analysis; (b) particle morphology analysis by transmission electron microscopy (TEM); (c) In vitro drug release profile of DOX and ET from DE-FPLN nanoparticles. The drug release study was performed by dialysis method in phosphate buffered saline $(\mathrm{pH} 7.4)$ and acetate buffered saline $(\mathrm{pH}$ 5.0). The drug release amount was quantified by HPLC method. ${ }^{*} p<0.05$ is the statistical difference between different conditions. 

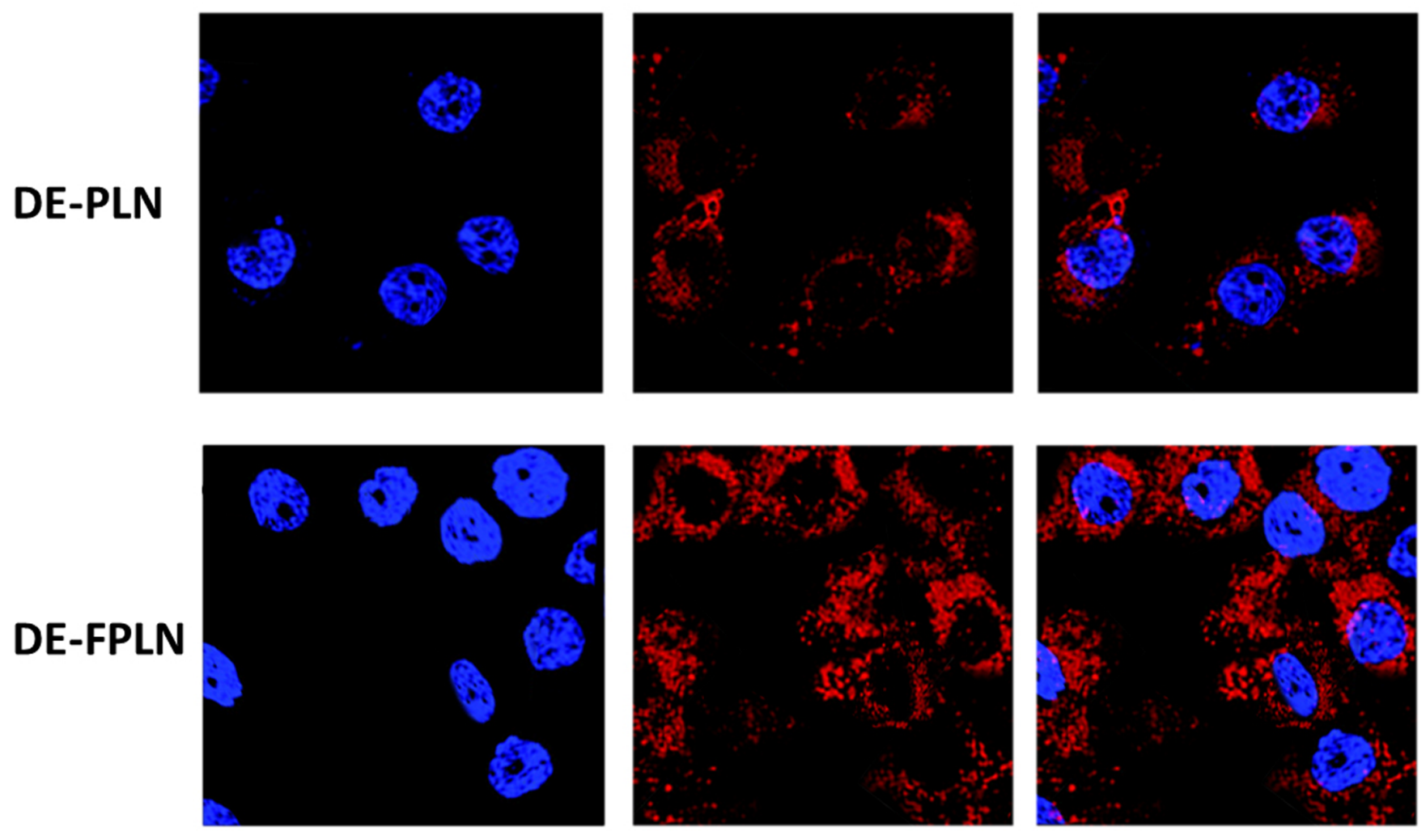

\section{Figure 3}

Cellular uptake analysis in Osteosarcoma. In vitro cellular uptake study in MG63 cancer cells. The distribution of DE-PLN and DE-FPLN in MG63 cancer cells was evaluated by confocal laser scanning microscopy using Nile Red as a fluorescent moiety.

(a)
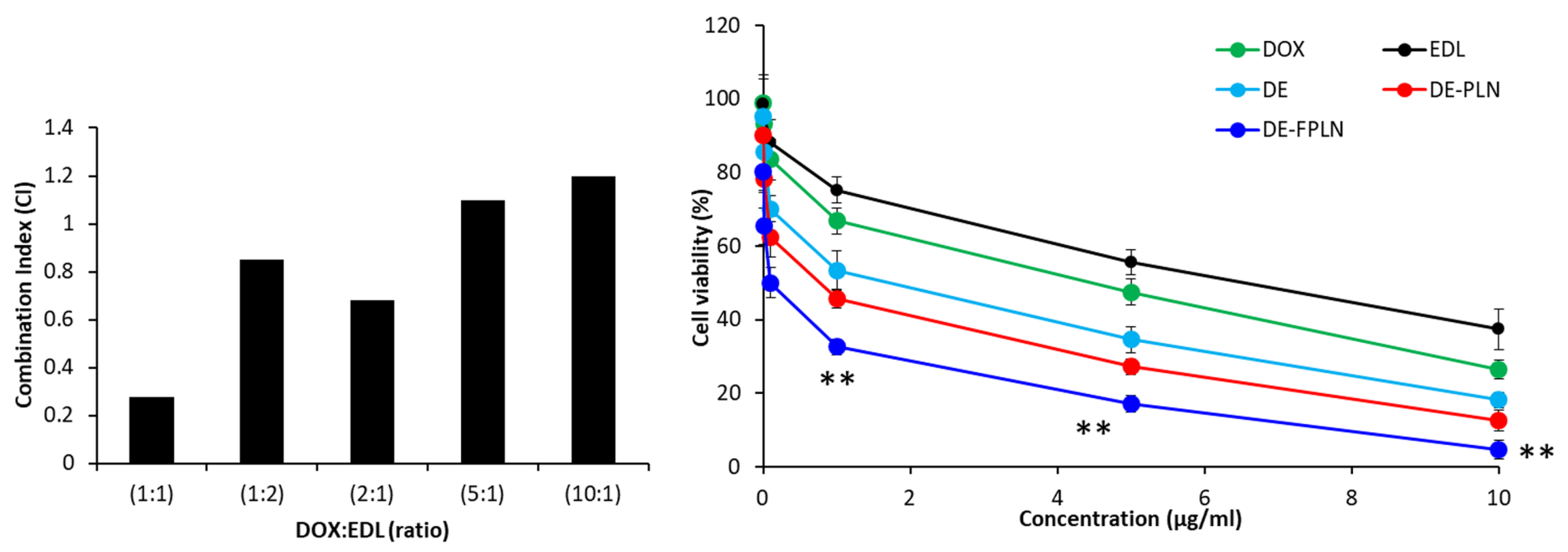

Figure 4 
In vitro anticancer effect of combination. (a) Combination index analysis of different ratiometric combinations of DOX and ET. The cell viability was determined and then fitted in Calcusyn Software; (b) cell viability analysis using MTS assay. A fixed ratio of 1:1 of DOX:ET was used to load in the nanoparticles. ${ }^{*} \mathrm{p}<0.001$ is the statistical difference between free DOX and DE-FPLN.
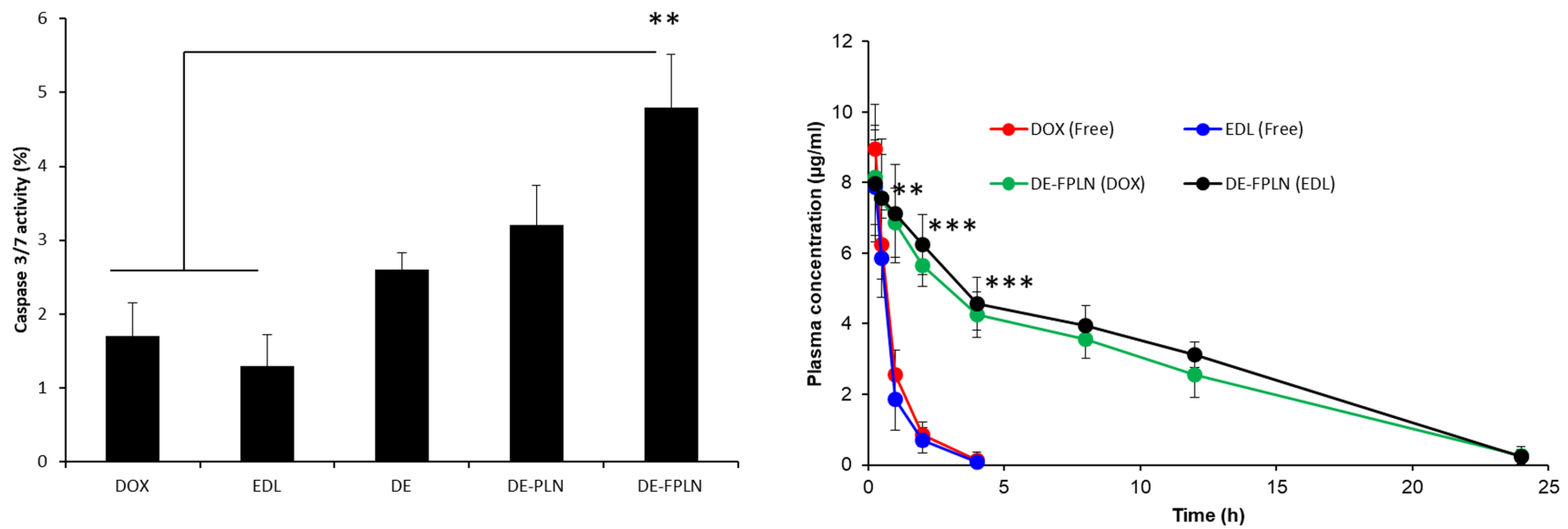

Figure 5

Apoptosis and pharmacokinetic analysis. (a) Caspase-3/7 activity analysis was performed by CaspaseGlo 3/7 luminescence assay after incubation for $24 \mathrm{~h}$. Different formulations of single and dual-drug were treated with MG63 cancer cells; (b) pharmacokinetic analysis of free DOX/EDL and DE-FPLN in animals. The formulations were administered intravenously and blood was collected in a timely manner. ${ }^{*} \mathrm{p}<0.001$ and ${ }^{* *} p<0.0001$ is the statistical difference between free DOX and DE-FPLN. 

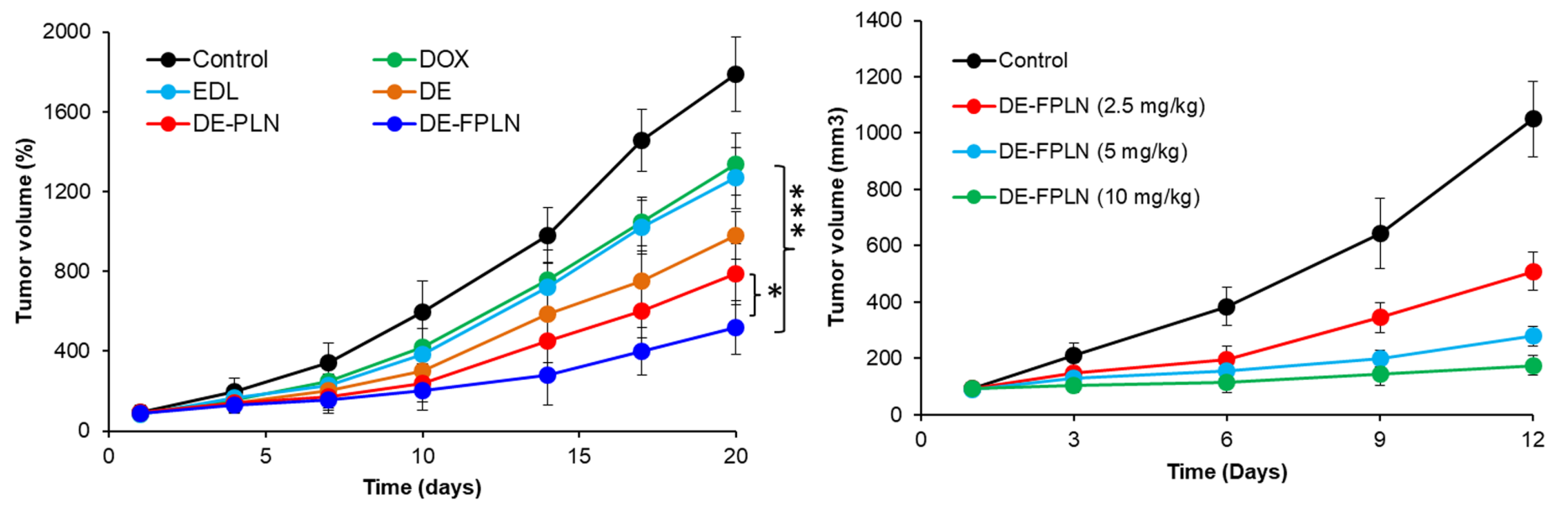

(c)

(d)
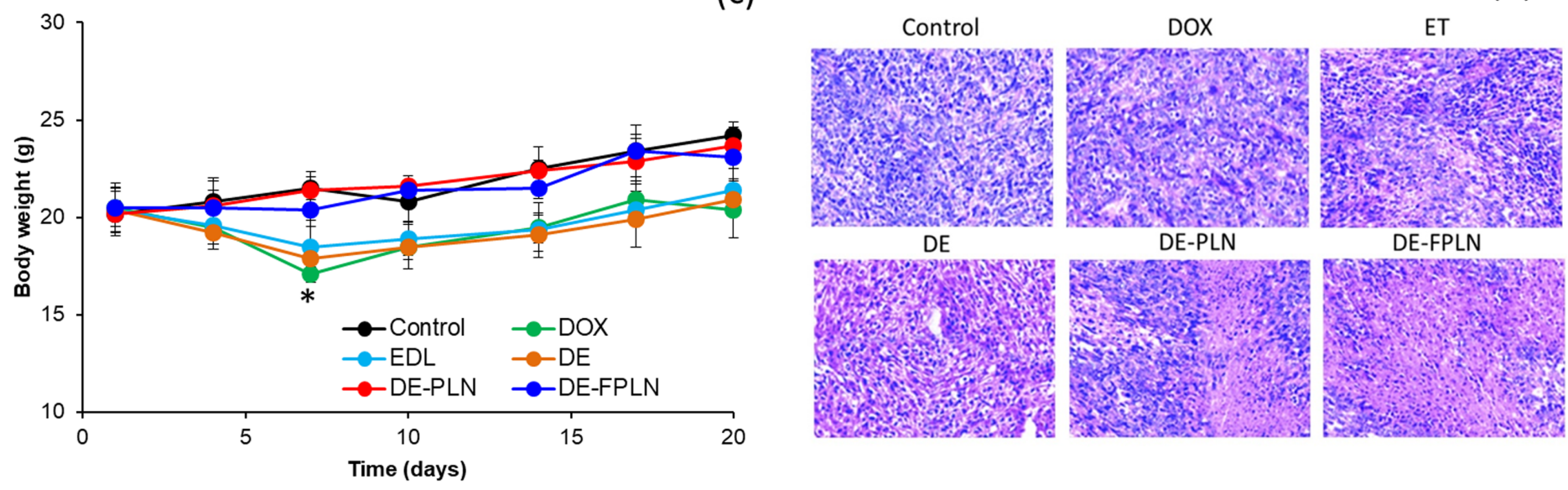

\section{Figure 6}

In vivo therapeutic efficacy analysis of combinational nanoparticles. (a) In vivo antitumor efficacy analysis in MG63 cancer cell bearing xenograft model. The tumor mice was administered with Doxorubicin (DOX), Edelfosine (EDL), Doxorubicin and Edelfosine cocktail (DE), DOX/EDL-loaded polymer lipid nanoparticles (DE-PLN), and DOX/EDL-loaded folate-conjugated polymer lipid nanoparticles (DEFPLN) and the tumor volumes were measured by vernier caliper. The free drug and formulations were administered at a dose of $5 \mathrm{mg} / \mathrm{kg}$; (b) tumor volume measurement following the different dose administration of DE-FPLN (2.5, 5, and $10 \mathrm{mg} / \mathrm{kg})$; (c) body weight analysis as a marker for toxicity patterns. The free drug and formulations were administered at a dose of $5 \mathrm{mg} / \mathrm{kg}$; (c) H\&E analysis of tumors extracted from different animal groups. The mice were sacrificed and tumors were extracted and subjected to H\&E analysis. ${ }^{*} p<0.05$ and ${ }^{* * *} p<0.001$ is the statistical difference between the indicated groups in the Figure 6.

Figure 7 
In vivo toxicity analysis in animal model. Acute toxicity analysis of free drugs and drug-loaded formulations in vital organs included heart, liver, spleen, lungs and kidney by H\&E staining analysis. The toxicity of DOX in heart is highlighted by arrow marks which showed disrupted cardiac muscle fibres and cytoplasmic vacuolization. Liver and kidney of DOX-treated group showed signs of marked degenerative changes.

\section{Supplementary Files}

This is a list of supplementary files associated with this preprint. Click to download.

- Supplementaryfile.docx 